\title{
Boundary Value Problems for Symmetric Positive Differential Operators of Odd Order*
}

\author{
MICHAEL G. CRANDALL
}

Communicated by Ralph PhILlips

Introduction. This paper treats a generalization to any odd order of the concept of first order symmetric positive systems of partial differential equations as introduced by K. O. Friedrichs [3]. In particular, we define concepts of weak and strong solutions of appropriate boundary value problems and show these concepts coincide (i.e., "weak = strong"). This result is then used to establish existence and uniqueness of strong solutions of our symmetric positive boundary value problems.

We set up the problem in the following way. Let $k \geqq 0$ be an integer. Let $\Omega$ be an open subset of $E^{p}$ (real $p$-dimensional Euclidean space) with a smooth boundary $\beta . H_{0}^{k}(\Omega)$ is the usual Hilbert space of complex $m$-vector-valued functions obtained by completing $C_{0}^{\infty}(\Omega)$ under the norm induced by the inner product

$$
(u, v)_{k}=\sum_{|\alpha| \leqq k}\left(D^{\alpha} u, D^{\alpha} v\right) \quad\left(u, v \varepsilon C_{0}^{\infty}(\Omega)\right)
$$

where $D^{\alpha}=D_{1}^{\alpha_{1}} \cdots D_{p}^{\alpha_{p}}=\left(\partial / \partial x_{1}\right)^{\alpha_{1}} \cdots\left(\partial / \partial x_{p}\right)^{\alpha_{p}},|\alpha|=\alpha_{1}+\cdots+\alpha_{p}$ and $(,$,$) is the \mathscr{L}_{2}(\Omega)$ inner product. Let $H_{0}^{-k}(\Omega)$ be the subset of distributions in $\Omega$ which have linear extensions from $C_{0}^{\infty}(\Omega)$ to $H_{0}^{k}(\Omega)$ which are continuous on $H_{0}^{k}(\Omega)$. With a suitable interpretation (see Sec. 1) $H_{0}^{-k}(\Omega)$ is the dual of $H_{0}^{k}(\Omega)$ with respect to an extension of the $\mathfrak{L}_{2}(\Omega)$ inner product, and given the topology induced by this identification it is a Hilbert space. Differential operators of order at most $2 k$ define bounded linear operators from $H_{0}^{k}(\Omega)$ into $H_{0}^{-k}(\Omega)$.

Now let

$$
L=\sum_{|\alpha| \leqq 2 k+1} A_{\alpha} D^{\alpha}
$$

be a differential expression of order $2 k+1$ where the $A_{\alpha}$ are smooth $m \times m$ matrix valued functions in $\Omega \cup \beta$. Let $N$ be a function mapping $x \varepsilon \beta$ into $N(x)$,

* This research is supported in part by the Office of Naval Research Nonr 222(62) and the National Science Foundation Grant GP 7458. 
a linear subspace of $C^{m} . N(x)$ is assumed to vary smoothly with $x$. We define $D(\mathrm{~L})$ to be the linear space consisting of all smooth functions $u$ such that (i) $u \varepsilon H_{0}^{k}(\Omega)$, (ii) $u$ has bounded support, (iii) the $k^{\text {th }}$ normal derivative of $u, D_{n}^{k} u$, satisfies $D_{n}^{k} u(x) \varepsilon N(x)$ for $x \varepsilon \beta$. For smooth functions with compact support (i) is a boundary condition equivalent to $D_{n}^{j} u(x)=0$ for $x \varepsilon \beta$ and $0 \leqq j \leqq k-1$. We define an operator $\mathrm{L}$ with domain $D(\mathrm{~L})$ by setting $\mathbf{L} u=L u$ for $u \varepsilon D(\mathbf{L})$ and regard $\mathrm{L}$ as a map L: $H_{0}^{k}(\Omega) \rightarrow H_{0}^{-k}(\Omega)$. $\mathrm{L}$ is always closable and its closure, $\overline{\mathrm{L}}$, is called the strong extension of $\mathrm{L}$. To define the weak extension we introduce the expression formally adjoint to $L$,

$$
L^{(*)}=\sum_{|\alpha| \leqq 2 k+1}(-1)^{|\alpha|} D^{\alpha} A_{\alpha}^{\prime},
$$

where $A_{\alpha}^{\prime}$ is the conjugate transpose of $A_{\alpha}$, and a domain $D\left(\mathrm{~L}^{(*)}\right)$ for $L^{(*)}$ to act on. $D\left(\mathrm{~L}^{(*)}\right)$ is the linear space of all smooth functions $v \varepsilon H_{0}^{k}(\Omega)$ with compact support satisfying

$$
(L u, v)-\left(u, L^{(*)} v\right)=0
$$

for all $u \varepsilon D(\mathrm{~L})$. It is easy to see $D\left(\mathrm{~L}^{(*)}\right)$ is determined by some $N^{*}(x)$ just as $D(\mathrm{~L})$ is by $N(x) . \mathrm{L}^{(*)}$ is the map $\mathrm{L}^{(*)}: H_{0}^{k}(\Omega) \rightarrow H_{0}^{-k}(\Omega)$ defined by $\mathrm{L}^{(*)} v=L^{(*)} v$ for $v \in D\left(L^{(*)}\right)$. The weak extension of $\mathrm{L}$ is the operator $\mathrm{L}^{(*) *}$ defined by $\mathbf{L}^{(*) *} w=f$ if $w \varepsilon H_{0}^{k}(\Omega), f \varepsilon H_{0}^{-k}(\Omega)$ and $(f, v)=\left(w, \mathbf{L}^{(*)} v\right)$ for all $v \varepsilon D\left(\mathbf{L}^{(*)}\right)$. It is trivial that $L^{(*) *} \supset \overline{\mathbf{L}}$. We show, provided $\beta$ is nowhere characteristic for $L$, that $\mathrm{L}^{(*) *}=\overline{\mathrm{L}}$, i.e., that "weak = strong".

In the event $k=0, H_{0}^{k}(\Omega)=H_{0}^{-k}(\Omega)=\mathfrak{L}_{2}(\Omega)$, and $\mathrm{L}^{(*) *}=\mathrm{L}^{(*) *}$, the usual Hilbert space adjoint of $L^{(*)}$. In this case "weak = strong" has been established by P. K. Lax and R. S. Phillips [5], H. O. Cordes [1] and L. Sarason [7]. The results of K. O. Friedrichs [2] include the case $N(x) \equiv C^{m}$ (no boundary condition) and also, in [3], he establishes "weak = strong" for symmetric positive systems (see below) by investigating the differentiability of solutions for such systems. Sarason [7] formulates the problem somewhat differently in order to treat inhomogeneous boundary conditions directly. A part of this work has been directed at the possibility of weakening assumptions on $\beta$ and $N(x)$, see also [6]. A generalization of the concept of first order symmetric positive equations is obtained in the framework of pseudo-differential operators by K. O. Friedrichs and P. D. Lax [4].

The result $\overline{\mathrm{L}}=\mathrm{L}^{(*) *}$ has immediate application to the case of symmetric positive systems as outlined below. (A clear and concise review of the case $k=0$ is given in [4, Section 1].) An expression

$$
L=\sum_{|\alpha| \leq 2 k+1} A_{\alpha} D^{\alpha}
$$

is called symmetric positive if $L+L^{(*)}$ is of order $2 k$ (i.e., $A_{\alpha}^{\prime}=A_{\alpha}$ for $|\alpha|=$ $2 k+1)$ and

$$
\left(\left(L+L^{(*)}\right) u, u\right) \geqq c(u, u)_{k}=c\|u\|_{k}^{2}
$$


for all $u \varepsilon C_{0}^{\infty}(\Omega)$ and some fixed $c>0$. Now by Green's formula, one can show that if $u$ vanishes up to order $k$ on $\beta$ and is smooth with compact support, then

$$
(L u, u)+(u, L u)=\left(\left(L+L^{(*)}\right) u, u\right)+\int_{\beta} D_{n}^{k} u \cdot B D_{n}^{k} u d \beta,
$$

where $B$ is a symmetric matrix. Similarly

$$
\left(v, L^{(*)} v\right)+\left(L^{(*)} v, v\right)=\left(\left(L+L^{(*)}\right) v, v\right)-\int_{\beta} D_{n}^{k} v \cdot B D_{n}^{k} v d \beta .
$$

Requiring $N(x)$ to be a maximal positive subspace of $C^{m}$ relative to the form

$$
\xi \rightarrow \xi \cdot B(x) \xi \quad\left(x \varepsilon \beta, \xi \varepsilon C^{m}\right)
$$

(dot is the usual $C^{m}$ inner product) guarantees that $N^{*}(x)$ is non-positive for the same form, as is easy to see. Hence, if $L$ is symmetric positive and $N(x)$ is maximal in the above sense, (1) and (2) yield

$$
\begin{aligned}
& (\mathrm{L} u, u)+(u, \mathrm{~L} u) \geqq c(u, u)_{k} \quad(u \varepsilon D(\mathbf{L})) \\
& \left(\mathrm{L}^{(*)} v, v\right)+\left(v, \mathrm{~L}^{(*)} v\right) \geqq c(v, v)_{k} \quad\left(v \varepsilon D\left(\mathrm{~L}^{(*)}\right)\right) .
\end{aligned}
$$

Applying the Schwartz inequality

$$
|(h, g)| \leqq\|h\|_{k}\|g\|_{-k},
$$

where $h \varepsilon H_{0}^{k}(\Omega)$ and $g \varepsilon H_{0}^{-k}(\Omega)$ and \|\|$_{i}$ is the norm in $H_{0}^{j}(\Omega)$, to (3) and (4) one sees that

$$
\begin{aligned}
& \|\mathbf{L} u\|_{-k} \geqq c\|u\|_{k} \quad(u \varepsilon D(\mathbf{L})) \\
& \left\|\mathbf{L}^{(*)} v\right\|_{-k} \geqq c\|v\|_{k} \quad\left(v \varepsilon D\left(\mathbf{L}^{(*)}\right)\right) .
\end{aligned}
$$

Inequality (5) implies $\overline{\mathrm{L}}$ is $1-1$ and inequality (6) implies $\mathrm{L}^{(*) *}$ is onto. Coupling these facts with $\mathrm{L}^{(*)}=\overline{\mathrm{L}}$ it follows that $\overline{\mathrm{L}}$ is a $1-1$ mapping of $H_{0}^{k}(\Omega)$ onto $H_{0}^{-k}(\Omega)$.

1. Preliminaries. All functions are assumed to have values in $C^{m}(m$-component complex vector space) unless it is stated otherwise. Let $\Omega$ be an open subset of $E^{p} . C_{0}^{\infty}(\Omega)$ has its usual meaning $D(\Omega)$ is $C_{0}^{\infty}(\Omega)$ equipped with the testing function topology, and $D^{\prime}(\Omega)$ is the set of all continuous linear functionals (distributions) on $\mathfrak{D}(\Omega)$. If $\varphi=\left(\varphi_{1}, \cdots, \varphi_{m}\right)$ and $\psi=\left(\psi_{1}, \cdots, \psi_{m}\right)$ are $C^{m}$ valued functions (or simply vectors in $C^{m}$ ) then

$$
\varphi \cdot \psi=\sum_{i=1}^{m} \varphi_{i} \bar{\psi}_{i}, \quad|\varphi|=(\varphi \cdot \varphi)^{1 / 2} .
$$

If $\varphi \cdot \psi$ is integrable over $\Omega$ then

$$
(\varphi, \psi)=\int_{\Omega} \varphi \cdot \psi
$$


If $\psi \varepsilon \mathfrak{R}_{1}^{100}(\Omega)$ (locally integrable functions) we define $f_{\psi} \varepsilon \mathfrak{D}^{\prime}(\Omega)$ by $f_{\psi}(\varphi)=(\varphi, \psi)$ for $\varphi \varepsilon D(\Omega)$. We do not distinguish between $\psi$ and $f_{\psi}$. In accordance with this notation we write the value of $f \varepsilon D^{\prime}(\Omega)$ at $\varphi \varepsilon D(\Omega)$ as $(\varphi, \bar{f})$. Then $(f, \varphi),(\varphi, f)$, etc., are given their natural meanings. We give some standard definitions and results.

Definition. Let $k$ be a non-negative integer. $H_{0}^{k}(\Omega)\left(H^{k}(\Omega)\right)$ is the Hilbert space obtained by completing $C_{0}^{\infty}(\Omega)\left(C_{0}^{\infty}(\bar{\Omega})\right)$ with respect to the norm induced by the inner product

$$
(\varphi, \psi)_{k}=\sum_{|\alpha| \leqq k}\left(D^{\alpha} \varphi, D^{\alpha} \psi\right) .
$$

Remark. $C_{0}^{\infty}(\bar{\Omega})$ is the set of functions obtained by restricting $C_{0}^{\infty}\left(E^{p}\right)$ functions to $\Omega$. In the obvious way we regard $H_{0}^{k}(\Omega)$ as a closed subspace of $H^{k}(\Omega)$ and $H^{k}(\Omega)$ as a subset of $\mathscr{L}_{2}(\Omega)$. If $\Omega_{1} \supset \Omega_{2}$ then we regard $H_{0}^{k}\left(\Omega_{2}\right)$ as a closed subspace of $H_{0}^{k}\left(\Omega_{1}\right)$. We note $H_{0}^{0}(\Omega)=H^{0}(\Omega)=\mathscr{L}_{2}(\Omega)$. We set $\|\varphi\|_{k}=(\varphi, \varphi)_{k}^{\frac{1}{k}}$ for $\varphi$ \& $H^{k}(\Omega)$. When necessary to avoid confusion these norms and inner products will be indexed by $\Omega, e . g .,(,)_{k, \Omega}$ or \|\|$_{k, \Omega}$.

Definition. Let $k \geqq 0$ be an integer. $H_{0}^{-k}(\Omega)$ is the set of all $f \varepsilon D^{\prime}(\Omega)$ such that

$$
\|f\|_{-k}=\sup \left\{|(f, \varphi)|: \varphi \varepsilon D(\Omega) \text { and }\|\varphi\|_{k}=1\right\}
$$

is finite. $H_{0}^{-k}(\Omega)$ is equipped with the norm (1.1).

Remark. $H_{0}^{-k}(\Omega)$ is a normed linear space. If $k=0$ the definitions of $H_{0}^{k}(\Omega)$ and $H_{0}^{-k}(\Omega)$ coincide via the embedding of $\mathcal{L}_{1}^{100}(\Omega)$ into $\mathfrak{D}^{\prime}(\Omega)$. Let $f \varepsilon \mathfrak{D}^{\prime}(\Omega)$. The linear form $\varphi \rightarrow(\varphi, f)$ defined for $\varphi \varepsilon C_{0}^{\infty}(\Omega)$ has a continuous linear extension to $H_{0}^{k}(\Omega)$ if and only if $f \varepsilon H_{0}^{-k}(\Omega)$. For such $f$ we denote the value of the extended form at $\varphi \varepsilon H_{0}^{k}(\Omega)$ by $(\varphi, f)$. Then $(f, \varphi),(\varphi, \bar{f})$, etc., are given their natural meanings for all $f \varepsilon H_{0}^{-k}(\Omega)$ and $\varphi \varepsilon H_{0}^{k}(\Omega)$. We note again the Schwartz inequality

$$
|(f, \psi)| \leqq\|f\|_{-k}\|\psi\|_{k} \quad\left(f \varepsilon H_{0}^{-k}(\Omega), \psi \varepsilon H_{0}^{k}(\Omega)\right) .
$$

Theorem 1.3. Every continuous linear functional on $H_{0}^{k}(\Omega)$ has the form $\varphi \rightarrow$ $(\varphi, f)$ for some fixed $f \varepsilon H_{0}^{-k}(\Omega)$, and every continuous linear functional on $H_{0}^{-k}(\Omega)$ has the form $f \rightarrow(f, \psi)$ for some fixed $\psi \varepsilon \varepsilon H_{0}^{k}(\Omega) . H_{0}^{-k}(\Omega)$ is a Hilbert space, i.e., it is $a$ Banach space under the norm defined in (1.1) and there is an inner product $(,)_{-k}$ such that $\|f\|_{-k}^{2}=(f, f)_{-k}$ for $f \varepsilon H_{0}^{-k}(\Omega)$.

Theorem 1.4. Let $l$ and $j$ be integers, where $j \geqq l$. Then

$$
D(\Omega) \subset H_{0}^{i}(\Omega) \subset H_{0}^{l}(\Omega) \subset D^{\prime}(\Omega)
$$

in the topological sense. Moreover $C_{0}^{\infty}(\Omega)$ is sequentially dense in each space listed.

As $H_{0}^{i}(\Omega) \subset D^{\prime}(\Omega)$ when $j$ is any integer we take $D^{\alpha} \varphi$, for $\varphi \varepsilon H_{0}^{i}(\Omega)$, in the sense of distributions. Now if $\varphi \varepsilon C^{\infty}(\Omega)$ and $L$ is any differential operator with coefficients in $\mathcal{L}_{1}^{\text {1oo }}(\Omega)$ then $L \varphi \varepsilon \&_{1}^{100}(\Omega) \subset D^{\prime}(\Omega)$. It could happen $L \varphi$ \& $H_{0}^{i}(\Omega)$ 
for some integer $j$. In the rest of this paper we will, unless otherwise specified, regard differential operators as initially defined on $C_{0}^{\infty}(\Omega)$ and then closed as maps between the Hilbert spaces under consideration. We remark that, in all cases considered here, this definition is unambiguous and gives back the usual action of the operator on smooth functions.

Let $C_{b}^{i}(\Omega), j \geqq 0$, be the set of functions possessing continuous and bounded derivatives in $\Omega$ of all orders through $j$.

Theorem 1.5. Let $L=\sum_{|\alpha| \leqq l} A_{\alpha} D^{\alpha}$ where $A_{\alpha} \varepsilon C_{b}^{\delta}(\Omega), \delta=\max (r-l, l)$. Then $\varphi \rightarrow L \varphi, \varphi \varepsilon C_{0}^{\infty}(\Omega)$, defines a bounded linear map of $H_{0}^{r}(\Omega)$ into $H_{0}^{r-l}(\Omega)$. If $l=2 k, r=k$, we have

$$
(L \varphi, \psi)=\left(\varphi, L^{(*)} \psi\right) \quad\left(\varphi, \psi \varepsilon H_{0}^{k}(\Omega)\right)
$$

and

$$
\|L \varphi\|_{-k} \leqq \text { cons. }\left(\sup \left|D^{\gamma} A_{\alpha}(x)\right|\right)\|\varphi\|_{k},
$$

where the sup is over $|\gamma| \leqq|\alpha| \leqq 2 k, x \varepsilon \Omega,\left|D^{\gamma} A_{\alpha}(x)\right|$ is any standard matrix norm, and the constant depends only $k, m$ and $p$.

Remark. We could weaken the smoothness assumptions on $A_{\alpha}$ in the above and, indeed, in the rest of the paper, but it does not seem worthwhile at this time.

We turn now to the point of this paper. Let $L=\sum_{|\alpha| \leqq 2 k+1} A_{\alpha} D^{\alpha}$ be a differential expression of order $2 k+1$. Here $k \geqq 0$ is an integer which will remain fixed for the rest of this paper, the $A_{\alpha}$ are $m \times m$ matrix valued functions, $A_{\alpha} \varepsilon C_{b}^{|\alpha|}(\bar{\Omega})$. Let $L^{(*)}$ be the formally adjoint expression,

$$
L^{(*)} \varphi=\sum_{|\alpha| \leqq 2 k+1}(-1)^{|\alpha|} D^{\alpha}\left(A_{\alpha}^{\prime} \varphi\right) \text { for } \varphi \varepsilon C_{0}^{\infty}(\Omega) .
$$

We specify $D(\mathrm{~L})$, a linear set of smooth functions with bounded support such that $H_{0}^{k}(\Omega) \supset D(\mathrm{~L}) \supset C_{0}^{\infty}(\Omega)$, and define a linear operator

$$
\text { L: } H_{0}^{k}(\Omega) \rightarrow H_{0}^{-k}(\Omega)
$$

by $\mathbf{L} u=L u$ for $u \varepsilon D(\mathrm{~L})$. Associated with $\mathrm{L}$ is the linear operator

$$
\mathrm{L}^{*}: H_{0}^{k}(\Omega) \rightarrow H_{0}^{-k}(\Omega)
$$

defined by $\mathbf{L}^{*} v=g$ if and only if $(\mathbf{L} u, v)=(u, g)$ for all $u \varepsilon D(\mathbf{L})$. More generally we define

Definition 1.6. Let $T$ be a linear operator

$$
\begin{aligned}
& T: H_{0}^{k}(\Omega) \rightarrow H_{0}^{-k}(\Omega) \\
& T: H_{0}^{k}(\Omega) \rightarrow H_{0}^{k}(\Omega) \\
& T: H_{0}^{-k}(\Omega) \rightarrow H_{0}^{-k}(\Omega) .
\end{aligned}
$$


with dense domain $D(T)$. Then $T^{*}$ is the linear operator

$$
\begin{aligned}
& T^{*}: H_{0}^{k}(\Omega) \rightarrow H_{0}^{-k}(\Omega) \\
& T^{*}: H_{0}^{-k}(\Omega) \rightarrow H_{0}^{-k}(\Omega) \\
& T^{*}: H_{0}^{k}(\Omega) \rightarrow H_{0}^{k}(\Omega),
\end{aligned}
$$

respectively, defined by $w \varepsilon D\left(T^{*}\right)$ and $T^{*} w=f$ if and only if $(T u, w)=(u, f)$ for all $u \varepsilon D(T)$.

Note that $T^{*}$ is the Hilbert space adjoint of $T$ where we regard $H_{0}^{k}(\Omega)$ and $H_{0}^{-k}(\Omega)$ as dual (rather than self-dual) and in analogy we have,

Theorem 1.7. Let $T$ be as in Definition 1.8, (i), (ii) or (iii). Then $T$ is closable if and only if $T^{*}$ is densely defined, and in this event $\bar{T}=T^{* *}$. If $T$ is bounded $T^{*}$ is everywhere defined, bounded, and the operator norms of $T$ and $T^{*}$ coincide. $T^{\prime \prime}$ is always closed.

With $\mathbf{L}$ as given above, it follows from Green's theorem that $L^{*} \supset \mathbf{L}_{0}^{(*)}$, where $\mathbf{L}_{0}^{(*)}$ is given by $L^{(*)}$ acting on $C_{0}^{\infty}(\Omega)$, so $\mathbf{L}$ is closable and $\bar{L}=\mathbf{L}^{* *}$. We now specify another linear set of smooth functions with bounded support $D\left(\mathrm{~L}^{(*)}\right)$, such that $D\left(\mathrm{~L}^{*}\right) \supset D\left(\mathrm{~L}^{(*)}\right) \supset C_{0}^{\infty}(\Omega)$. The operator $\mathrm{L}^{(*)}: H_{0}^{k}(\Omega) \rightarrow$ $H_{0}^{-k}(\Omega)$ is given by $L^{(*)}$ acting on $D\left(\mathrm{~L}^{(*)}\right)$. Then $\mathrm{L}^{*} \supset \mathrm{L}^{(*)}$ is clear. We call $\mathbf{L}^{(*) *}$ the weak extension of $\mathbf{L}$ (relative to $D\left(\mathbf{L}^{(*)}\right)$ ) and abbreviate it $\mathbf{L}_{w}$. The strong extension of $L$ is $\overline{\mathrm{L}}$, and we write $\mathrm{L}_{s}=\overline{\mathrm{L}}$. From $\mathrm{L}^{*} \supset \mathrm{L}^{(*)}$ and $\mathrm{L}^{* *}=\mathrm{L}_{s}$ follows $\mathrm{L}_{w} \supset \mathrm{L}_{s}$. We prove

Theorem I. Let $\Omega$ be an open subset of $E^{p}$ of class $C^{2 k+2}$. Let $\beta$ be the boundary of $\Omega$. Let

$$
L=\sum_{|\alpha| \leqq 2 k+1} A_{\alpha} D^{\alpha}
$$

where $A_{\alpha} \varepsilon C_{b}^{|\alpha|}(\bar{\Omega})$. Let $\beta$ be nowhere characteristic for L. For $x \varepsilon \beta$, let $N(x)$ be a linear subspace of $C^{m}$ which has constant dimension on each component of $\beta$ and has, locally, a $C^{2 k+1}$ basis. Let $D(\mathrm{~L})$ (resp. $D\left(\mathrm{~L}^{(*)}\right)$ ) be the linear set of smooth functions with compact support which belong to $H_{0}^{k}(\Omega)$ and satisfy $D_{n}^{k} u(x)$ \& $N(x)$ (resp. $D_{p}^{k} u(x) \varepsilon N^{*}(x)$ ), $x \varepsilon \beta$. Here $D_{n}$ is differentiation in the direction of the exterior normal to $\beta, N^{*}(x)=(B(x) N(x))^{\perp}$ (the orthogonal complement of $B(x) N(x)$ in $C^{m}$ ) and

$$
B(x)=(-1)^{k} \sum_{|\alpha|=2 k+1} n(x)^{\alpha} A_{\alpha}(x) .
$$

In equation (1.8), $n=\left(n_{1}, \cdots, n_{p}\right)$ is the exterior normal to $\beta, n^{\alpha}=n_{1}^{\alpha_{1}} \cdots n_{p}^{\alpha_{p}}$. Then $\mathrm{L}_{\boldsymbol{s}}=\mathrm{L}_{w}$.

We note that $D\left(\mathrm{~L}^{(*)}\right)$ consists of functions $v$ for which $(\mathrm{L} u, v)=\left(u, L^{(*)} v\right)$ for all $u \varepsilon D(\mathrm{~L})$. This follows from the formula

$$
(L u, v)-\left(u, L^{(*)} v\right)=\int_{\beta} B(x) D_{n}^{k} u(x) \cdot D_{n}^{k} v(x) d \beta
$$


which holds for smooth functions $u, v$ with compact support such that $D_{n}^{i} v(x)=$ $D_{n}^{i} u(x)=0$ for $x$ \& $\beta, 0 \leqq j \leqq k-1$.

As will be seen below, Theorem I is local in the sense it suffices to show that $u \varepsilon D\left(\mathrm{~L}_{w}\right) \Rightarrow u \varepsilon D\left(\mathrm{~L}_{s}\right)$ where the support of $u$ may be taken arbitrarily small. For this reason it is sufficient to prove the theorem in the special cases where $\Omega$ is either $E^{p}$ or a half-space, since by assumption every point in $\Omega \cup \beta$ has a neighborhood in $\Omega \cup \beta$ which is $C^{2 k+2}$ equivalent to an open ball in $E^{p}$ or the intersection of a ball and a closed half-space. This is not a proof of the claim just made, but the details are not hard. Hence we treat just the cases of $E^{p}$ and a half-space.

\section{Proof of Theorem I if $\Omega=E^{p}$.}

Theorem 2.1. Let $L=\sum_{|\alpha| \leq 2 k+1} A_{\alpha} D^{\alpha}$ satisfy the assumptions of Theorem I with $\Omega=E^{p}$. Let $D(\mathrm{~L})=D\left(\mathbf{L}^{(*)}\right)=C_{\mathrm{o}}^{\infty}\left(E^{p}\right)$. Then $\mathrm{L}_{w}=\mathrm{L}_{\mathbf{s}}$.

Proof. We have only to show that $u \varepsilon D\left(\mathrm{~L}_{w}\right)$ implies $u \varepsilon D\left(\mathrm{~L}_{s}\right)$, i.e., the pair $\left\{u, \mathbf{L}_{w} u\right\}$ lies in the closure of the graph of $\mathbf{L}$ in the Hilbert space $H_{0}^{k}\left(E^{p}\right) \times H_{0}^{-k}\left(E^{p}\right)$. This fact is easy to establish using well-known arguments. First, we may assume $u \varepsilon D\left(\mathrm{~L}_{w}\right)$ and $u$ has compact support.

For, if $u$ does not have compact support, choose a real-valued function $w_{\varepsilon} C_{0}^{\infty}\left(E^{p}\right)$ which is identically 1 in some neighborhood of the origin, and form $w_{\delta}(x)=w(\delta x)$ for $0<\delta<1$. Then $u_{\delta}=w_{\delta} u$ lies in $D\left(\mathbf{L}_{w}\right)$ and satisfies

$$
\mathbf{L}_{w} u_{\delta}=w_{\delta} L_{w} u+\left(L w_{\delta}-w_{\delta} L\right) u \text {. }
$$

Note $\left[L, w_{\delta}\right]=L w_{\delta}-w_{\delta} L$ is a differential operator of order $2 k$ whose coefficients (with sufficiently many of their derivatives) tend to zero with $\delta$. Thus, in virtue of Theorem 1.5, $\left[L, w_{\delta}\right]$ is a family of bounded operators from $H_{0}^{k}\left(E^{p}\right)$ to $H_{0}^{-k}\left(E^{p}\right)$ whose norms tend to zero with $\delta$. We show $u_{\delta} \varepsilon D\left(\mathbf{L}_{w}\right)$. By assumption, $\left(u, \mathbf{L}^{(*)} v\right)$ $=\left(\mathrm{L}_{w} u, v\right)$ for $v \varepsilon C_{0}^{\infty}\left(E^{p}\right)$. We find then

$$
\left(w_{\delta} u, \mathbf{L}^{(*)} v\right)=\left(u,\left(w_{\delta} L^{(*)}-L^{(*)} w_{\delta}\right) v\right)+\left(\mathbf{L}_{w} u, w_{\delta} v\right) \quad\left(v \varepsilon C_{0}^{\infty}\left(E^{p}\right)\right) .
$$

Now we observe that $\left(w_{\delta} L^{(*)}-L^{(*)} w_{\delta}\right)^{*}=T_{\delta}$ is everywhere defined, and $T_{\delta}=\left(L w_{\delta}-w_{\delta} L\right)$, as follows from Theorems 1.5 and 1.7. We see (2.2) implies $u_{\delta}=w_{\delta} u \varepsilon D\left(L_{w}\right)$ and $\mathbf{L}_{w} u_{\delta}=T_{\delta} u+w_{\delta} L_{w} u$. We now note the mapping $\delta \rightarrow w_{\delta} \varphi$, $0<\delta<1$, is continuous as a map of $H_{0}^{i}\left(E^{p}\right)$ into $H_{0}^{i}\left(E^{p}\right)$ for any integer $j$ and the corresponding operator norm is bounded uniformly in $\delta, 0<\delta<1$. Since $w_{\delta} \varphi=\varphi$ for all $\delta$ sufficiently small and fixed $\varphi \varepsilon C_{0}^{\infty}\left(E^{p}\right)$, we find $\lim _{\delta \rightarrow 0}\left\|w_{\delta} \varphi-\varphi\right\|_{i}$ $=0$ for all $\varphi \varepsilon H_{0}^{i}\left(E^{p}\right)$ and any integer $j$. We conclude

$$
\begin{aligned}
\lim _{\delta \rightarrow 0}\left(\| w_{\delta} u\right. & \left.-u\left\|_{k}+\right\| \mathbf{L}_{w} w_{\delta} u-\mathbf{L}_{w} u \|_{-k}\right) \\
& \leqq \lim _{\delta \rightarrow 0}\left(\left\|u-w_{\delta} u\right\|_{k}+\left\|\mathbf{L}_{w} u-w_{\delta} \mathbf{L}_{w} u\right\|_{-k}+\left\|T_{\delta} u\right\|_{-k}\right)=0 .
\end{aligned}
$$

It follows that $u \varepsilon D\left(\mathrm{~L}_{w}\right)$ if and only if $w_{\delta} u \varepsilon D\left(\mathrm{~L}_{w}\right) 0<\delta<1$. 
The rest of the proof of Theorem 2.1 is a standard argument using Friedrichs' mollifiers. We give a definition and list some properties important here, however, the reader is assumed to be familiar with the basic properties of mollifiers.

Let $\chi$ be a real-valued function, $\chi \varepsilon C_{0}^{\infty}\left(E^{p}\right)$. For $\varphi \varepsilon \&_{1}^{\text {loc }}\left(E^{p}\right)$ we define $\chi * \varphi$ by

$$
\chi * \varphi(x)=\int_{E^{\nu}} \chi\left(x-x^{\prime}\right) \varphi\left(x^{\prime}\right) d x^{\prime} .
$$

If $f \varepsilon \mathscr{D}^{\prime}\left(E^{p}\right)$ we define $\chi * f \varepsilon \mathscr{D}^{\prime}\left(E^{p}\right)$ by

$$
(\varphi, \chi * f)=\left(\check{\chi}^{*} \varphi, f\right) \quad\left(\varphi \varepsilon C_{0}^{\infty}\left(E^{p}\right)\right),
$$

where $\check{\chi}\left(x_{1}, \cdots, x_{p}\right)=\chi\left(-x_{1}, \cdots,-x_{p}\right)$. These definitions coincide if $f \varepsilon$ $\mathcal{L}_{1}^{\text {loo }}\left(E^{\nu}\right)$. Then $D^{\alpha}(\chi * f)=\left(D^{\alpha} \chi\right) * f=\chi *\left(D^{\alpha} f\right)$ for $f \varepsilon D^{\prime}\left(E^{p}\right)$ and $\chi * \varphi \varepsilon$ $H_{0}^{l}\left(E^{p}\right)$ for all $l$ if $\varphi \varepsilon H_{0}^{i}\left(E^{p}\right)$ for some $j$. Let $\chi_{e}, 0<\epsilon<1$, be defined by $\chi_{\epsilon}(x)=$ $\epsilon^{-p} \chi(x / \epsilon)$.

Lemma 2.3 (Friedrichs). Let $A$ be an $m \times m$ matrix, $A \varepsilon C_{b}^{1}\left(\mathrm{E}^{p}\right)$. Then there is a constant such that for $1 \leqq i \leqq p$ and $\varphi \varepsilon \mathfrak{L}_{2}\left(E^{p}\right)$

(i) $\left\|D_{i}\left(A\left(\chi_{\epsilon} * \varphi\right)-\chi_{\epsilon} *(A \varphi)\right)\right\|_{0} \leqq$ cons. $\|\varphi\|_{0}$.

The constant may be chosen independent of $\varphi$ and $\epsilon, 0<\epsilon<1$. Moreover, for $\varphi \varepsilon \mathfrak{L}_{2}\left(E^{p}\right)$,

$$
\lim _{\epsilon \rightarrow 0}\left\|D_{i}\left(A\left(\chi_{\epsilon} * \varphi\right)-\chi_{e} *(A \varphi)\right)\right\|_{0}=0 .
$$

The proof is standard and is omitted.

Corollary 2.4. Let $A$ be an $m \times m$ matrix-valued function, $A$ \& $C_{b}^{|\alpha|}\left(E^{p p}\right)$, where $\alpha$ is a multi-index, $|\alpha| \leqq 2 k+1$. Let $\varphi \varepsilon H_{0}^{k}\left(E^{p}\right)$. Then $D^{\alpha}\left(A\left(\chi_{\mathrm{e}}^{*} \varphi\right)\right)$ and $D^{\alpha}\left(\chi_{\mathrm{e}} *(A \varphi)\right)$ are in $H_{0}^{-k}\left(E^{p}\right)$. Moreover the maps $T_{\mathrm{e}}: H_{0}^{k}\left(E^{p}\right) \rightarrow H_{0}^{-k}\left(E^{p}\right)$ defined by

$$
\left.T_{\mathrm{e} \varphi}=D^{\alpha}\left(A\left(\chi_{\mathrm{e}} * \varphi\right)\right)-\chi_{\mathrm{e}} *(A \varphi)\right)
$$

are uniformly bounded in $\epsilon, 0<\epsilon<1$, and $\lim _{\epsilon \rightarrow 0} T_{\iota}=0$ strongly.

The proof of this lemma is a little messy due to notation, but it is only an excercise in integration by parts and application of Friedrichs' lemma, so we omit it.

Now we specialize $\chi$ to our purpose. Let $j$ be a real-valued function of one real variable with the properties

$$
\begin{aligned}
& \text { (i) } j \varepsilon C_{0}^{\infty}(E), \quad j(y)=0 \text { for }|y|>1 \text {, } \\
& \text { (ii) } j \text { is even, } \\
& \text { (iii) } \int_{-\infty}^{\infty} j(y) d y=1, \\
& \text { (iv) } j \geqq 0 .
\end{aligned}
$$


Set $\chi\left(x_{1}, \cdots, x_{n}\right)=j\left(x_{1}\right) \cdots j\left(x_{n}\right)$ and set $J_{\epsilon} f=\chi_{\epsilon} * f$ for $f \varepsilon D^{\prime}\left(E^{p}\right) . J_{\epsilon}$ is called a mollifier. $J_{\epsilon}$ commutes with differentiation, multiplication by constant coefficient matrices, and is symmetric in the sense $\left(J_{\epsilon} \varphi, \psi\right)=\left(\varphi, J_{\epsilon} \psi\right)$ for $\varphi \varepsilon H_{0}^{i}\left(E^{p}\right), \psi \varepsilon H_{0}^{-i}\left(E^{p}\right)$. If $\varphi$ is continuous and has compact support $J_{\epsilon} \varphi$ tends uniformly to $\varphi$ as $\epsilon \rightarrow 0$. We have

Lemma 2.6. Let $j$ be any integer and $f \varepsilon H_{0}^{i}\left(E^{p}\right)$. Then $J_{\epsilon} f \varepsilon H_{0}^{i}\left(E^{p}\right)$ and

$$
\begin{gathered}
\left\|J_{\epsilon} f\right\|_{i} \leqq\|f\|_{j} \\
\lim _{\epsilon \rightarrow 0}\left\|J_{\epsilon} f-f\right\|_{i}=0 .
\end{gathered}
$$

The proofs for $j \geqq 0$ are classical, and for $j<0$ the lemma follows from the symmetry $\left(J_{\epsilon} \varphi, \psi\right)=\left(\varphi, J_{\epsilon} \psi\right)$ for $\varphi \varepsilon H_{0}^{-i}\left(E^{p}\right), \psi \varepsilon H_{0}^{j}\left(E^{p}\right)$ and the case $j \geqq 0$.

End of the proof of Theorem 2.1. Let $u \varepsilon D\left(\mathrm{~L}_{w}\right)$ and the support of $u$ be compact. Then $u_{\epsilon}=J_{\epsilon} u$ is in $C_{0}^{\infty}\left(E^{p}\right)=D(\mathrm{~L})$. For $\psi \varepsilon C_{0}^{\infty}\left(E^{p}\right)$

$$
\begin{aligned}
\left(\mathbf{L}_{\epsilon}, \psi\right) & =\left(u,\left(J_{\epsilon} L^{(*)}-L^{(*)} J_{\epsilon}\right) \psi\right)+\left(u, \mathbf{L}^{(*)} J_{\epsilon} \psi\right) \\
& =\left(u,\left(J_{\epsilon} L^{(*)}-L^{(*)} J_{\epsilon}\right) \psi\right)+\left(J_{\epsilon} \mathbf{L}_{w} u, \psi\right) .
\end{aligned}
$$

Let $T_{\epsilon}$ be given by $\left[J_{\epsilon}, L^{(*)}\right]=J_{\epsilon} L^{(*)}-L^{(*)} J_{\epsilon}$ acting on $C_{0}^{\infty}\left(E^{p}\right)$. We regard $T_{\epsilon}$ as a map with domain in $H_{0}^{k}\left(E^{p}\right)$ and range in $H_{0}^{-k}\left(E^{p}\right)$. In virtue of Corollary 2.4, $T_{\epsilon}$ is bounded uniformly in $\epsilon, 0<\epsilon<1$. Thus $T^{\text {* }}$ is everywhere defined on $H_{0}^{k}\left(E^{p}\right)$ and uniformly bounded in $\epsilon, 0<\epsilon<1$, by Theorem 1.7. We have

$$
\mathrm{L} u_{\epsilon}=T^{*}{ }_{\epsilon}^{*} u+J_{\epsilon} \mathrm{L}_{w} u \text {. }
$$

Now $\lim _{\epsilon \rightarrow 0}\left\|T_{\epsilon}^{*} \varphi\right\|_{-k}=0$ for $\varphi \varepsilon C_{0}^{\infty}\left(E^{p}\right)$. In fact $T^{*}{ }_{\epsilon}^{*} \varphi$ tends uniformly to zero (as a function) with $\epsilon$ for $\varphi \varepsilon C_{0}^{\infty}\left(E^{p}\right)$, and has its support in some compact set independent of $\epsilon, 0<\epsilon<1$. It follows that $\lim _{\epsilon \rightarrow 0} T_{\epsilon}^{*}=0$ strongly. Combining these observations with Lemma 2.6 we find

$$
\begin{aligned}
\lim _{\epsilon \rightarrow 0}\left\|u_{\epsilon}-u\right\|_{k}+\| \mathbf{L} u_{\epsilon} & -\mathbf{L}_{w} u \|_{-k} \\
& \leqq \lim _{\epsilon \rightarrow 0}\left\|u-J_{\epsilon} u\right\|_{k}+\left\|T_{\epsilon}^{*} u\right\|_{-k}+\left\|\mathbf{L}_{w} u-J_{\epsilon} \mathbf{L}_{w} u\right\|_{-k} \\
& =0 .
\end{aligned}
$$

It follows that $\left\{u, \mathbf{L}_{w} u\right\}$ lies in the closure of the graph of $\mathrm{L}$, and proof of Theorem 2.1 is complete.

3. Theorem I when $\Omega$ is a half-space. Let

$$
S=\left\{\left(x_{0}, x_{1}, \cdots, x_{p-1}\right): x_{0}<0, x_{i} \varepsilon E^{1}, \quad 1 \leqq j \leqq p-1\right\} .
$$

We write elements of $E^{p}$ in the form $\left(x_{0}, x\right)$ with $x_{0} \varepsilon E$ and $x=\left(x_{1}, \cdots, x_{p-1}\right) \varepsilon$ $E^{p-1}$. If $\alpha$ is a multi-index we write $\alpha=\left(\alpha_{0}, \delta\right)$ with $\alpha_{0}$ a non-negative integer and $\delta=\left(\alpha_{1}, \cdots, \alpha_{p-1}\right)$ a multi-index. We set $D^{\alpha}=D_{0}^{\alpha_{0}} D^{\delta}$ with $D_{0}^{\alpha_{0}}=\left(\partial / \partial x_{0}\right)^{\alpha \bullet}$, $D^{\delta}=D_{1}^{\alpha_{1}} \cdots D_{p-1}^{\alpha_{p-1}}=\left(\partial / \partial x_{1}\right)^{\alpha_{1} \cdots}\left(\partial / \partial x_{p-1}\right)^{\alpha_{p-1}}$. 
Theorem 3.1. Theorem $I$ is true in the case $\Omega=S$.

We adopt the notation and assumptions of Theorem I setting $\Omega=S$, and also setting $A_{(2 k+1,0, \cdots, 0)}=A$, i.e.,

$$
L=\sum A_{\alpha} D^{\alpha}=A D_{0}^{2 k+1}+\sum_{\alpha_{0}<2 k+1} A_{\alpha} D^{\alpha} .
$$

Above and hereafter, $\sum$ stands for a summation over only those $\alpha$ such that $|\alpha| \leqq 2 k+1$. In this case the matrix $B$ of (1.8) is a function of $x \varepsilon E^{p-1}, B(x)=$ $(-1)^{k} A(0, x)$.

Proof of Theorem 3.1. It is obvious that $D(\mathrm{~L})$ and $D\left(\mathrm{~L}^{(*)}\right)$ are local in the sense that they are invariant under multiplication by scalar-valued functions in $C_{0}^{\infty}\left(E^{p}\right)$. It then follows from the boundedness of differential opertors of order not exceeding $2 k$ that $D\left(\mathrm{~L}_{w}\right)$ and $D\left(\mathrm{~L}_{s}\right)$ are also local. We must show $u \varepsilon D\left(\mathbf{L}_{w}\right)$ implies $u \varepsilon D\left(\mathrm{~L}_{s}\right)$. The local nature of $D\left(\mathrm{~L}_{w}\right)$ allows us to repeat the argument used in the proof of Theorem 3.1 and assume $u$ has compact support. We now introduce a partition of unity to localize the problem further. Let $\left\{\mathcal{O}_{i}\right\}_{1}^{\infty}$ be a sequence of real-valued functions such that $\sum_{i} \mathcal{O}_{i} \equiv 1$, where $\left\{\mathcal{O}_{i}\right\}_{1}^{\infty} \subset C_{0}^{\infty}\left(E^{p}\right)$, and the set of supports of the $\mathcal{O}_{i}$ is locally finite. Then $u=\sum_{i} \mathcal{O}_{i} u=\sum_{i} u_{2}$, with $u_{i}=\mathcal{O}_{i} u$, and the sum is finite since the support of $u$ is compact. Now $u_{i} \varepsilon D\left(\mathrm{~L}_{w}\right)$ for all $i$ and if $u_{i} \varepsilon D\left(\mathrm{~L}_{s}\right)$ for all $i$ then $u \varepsilon D\left(\mathrm{~L}_{s}\right)$ follows. If $u_{i}$ has compact support contained in $S$ we may use the argument of Theorem 2.1. In this case $J_{\epsilon}$ will not leave $H_{0}^{k}(S)$ invariant (regarding $J_{\epsilon}$ as a map with domain and range in $\left.H_{0}^{k}\left(E^{p}\right) \supset H_{0}^{k}(S)\right)$ so we use instead $\mathcal{O} J_{\epsilon}$ where $\mathcal{O} \varepsilon C_{0}^{\infty}(S)$ and $\theta$ is identically 1 in some neighborhood of the support of $u_{i}$. Then setting $u_{i \epsilon}=\mathcal{O} J_{\epsilon} u_{i}$ we have $u_{i \epsilon} \varepsilon D(\mathbf{L}), \lim _{\epsilon \rightarrow 0}\left\|\mathbf{L} u_{i \epsilon}-\mathbf{L}_{w} u_{i}\right\|_{-k, s}=0$, and $\lim _{\epsilon \rightarrow 0} \| u_{i \epsilon}-$ $u_{i} \|_{k}=0$. The proof of these assertions is a simple modification of the argument used in proving Theorem 2.1, and is omitted. It remains to treat the following case: $u \varepsilon D\left(\mathrm{~L}_{w}\right)$ and the support of $u$ (which may be taken small) intersects the hyperplane $x_{0}=0$.

We make some standard algebraic simplifications following Lax-Phillips [5]. Let $L, D(\mathrm{~L}), D\left(\mathrm{~L}^{(*)}\right)$ be given as in Theorem I. Let $\gamma$ be an $m \times m$ matrix function with a uniformly bounded inverse, $\gamma \varepsilon C_{b}^{2 k+1}(\bar{\Omega})$.

Lemma 3.2. Set $L_{1}=\gamma L, D\left(\mathrm{~L}_{1}\right)=D(\mathrm{~L})$ and $D\left(\mathrm{~L}_{1}^{(*)}\right)=\gamma^{-1} D\left(\mathrm{~L}^{(*)}\right)$. Then $\mathrm{L}_{s} u=f$ if and only if $\mathrm{L}_{1 s} u=\gamma f$, and $\mathrm{L}_{w} u=f$ if and only if $\mathrm{L}_{1 w} u=\gamma f$.

Lemma 3.3. Let $\kappa$ have the properties listed for $\gamma$ above. Set $L_{1}={ }_{\kappa} L_{\kappa}{ }^{-1}$. Let $D\left(\mathbf{L}_{1}\right)=\kappa D(\mathbf{L})$ and $D\left(\mathbf{L}_{1}^{(*)}\right)=\left(\kappa^{\prime}\right)^{-1} D\left(\mathbf{L}^{(*)}\right)$. Then $\mathbf{L}_{s} u=f$ if and only if $\mathbf{L}_{1 s}(\kappa u)=$ $\kappa f$ and $\mathrm{L}_{w} u=f$ if and only if $\mathrm{L}_{1 w}(\kappa u)=\kappa f$.

The proofs are trivial and are omitted.

Now assume $u \varepsilon D\left(\mathrm{~L}_{w}\right)$ and the support of $u$, which we hereafter denote by $\sigma(u)$, intersects the hyperplane $x_{0}=0$. By taking $\sigma(u)$ small we may assume $A$ (the coefficient of $D_{0}^{2 k+1}$ in $L$ ) has a uniformly bounded inverse on $\sigma(u)$. (Recall $x_{0}=0$ is nowhere characteristic for $L$ by assumption.) We multiply $L$ on the left 
by a smooth invertible matrix which agrees with $A^{-1}$ in a neighborhood of $\sigma(u)$ and get a new differential expression and an equivalent problem as in Lemma 3.2. Since the behavior of the coefficients of $L$ off of $\sigma(u)$ does not affect the validity of the statements $u \varepsilon D\left(\mathbf{L}_{s}\right)$ or $u \varepsilon D\left(\mathbf{L}_{w}\right)$, we may now assume the coefficient of $D_{0}^{2 k+1}$ in $L$ is the identity matrix. Doing so, we proceed as in Lemma 3.3. We take $\sigma(u)$ so small that $\kappa$ may be chosen to satisfy

$\kappa(0, x) N(x)=\left\{\rho: \rho=\left(\rho_{1}, \cdots, \rho_{m}\right) \varepsilon C^{m}\right.$ and $\rho_{i}=0$ for $\left.r<j \leqq m\right\}$, where $r$ is the dimension of $N(x)$, for all $x$ in a neighborhood of $\sigma(u) \cap\left\{\left(x_{0}, x\right)\right.$ : $\left.x_{0}=0\right\}$ projected into $E^{p-1}$. The existence of such a $\kappa$ is guaranteed by our assumptions on $N(x)$.

We have reduced the problem to the special form

$$
\begin{gathered}
L=D_{0}^{2 k+1}+\sum_{\alpha_{0}<2 k+1} A_{\alpha} D^{\alpha} \\
D(\mathbf{L})=\left\{\varphi: \varphi \varepsilon C_{0}^{2 k+1}(\bar{S}) \text { and } D_{0}^{i} \varphi(0, x)=0,\right. \\
\left.0 \leqq j \leqq k-1, D_{0}^{k} \varphi_{i}(0, x)=0, r<i \leqq m\right\} . \\
D\left(\mathbf{L}^{(*)}\right)=\left\{v: v \varepsilon C_{0}^{2 k+1}(\bar{S}) \text { and } D_{0}^{i} v(0, x)=0,\right. \\
\left.0 \leqq j \leqq k-1, D_{0}^{k} v_{i}(0, x)=0,1 \leqq i \leqq r\right\} .
\end{gathered}
$$

We now generalize Lemma 1.1 of Lax-Phillips [5] to our case. Let $W$ be a measurable subset of $E^{a}$ and $X$ be a Banach space. $\mathscr{L}_{2}(W, X)$ is the Banach space of square integrable (with respect to the usual Lebesque measure) functions $f$ mapping $W$ into $X$.

Lemma 3.6. Let $u \varepsilon D\left(\mathbf{L}_{w}\right)$ where $L$ is given by (3.4), $D(\mathbf{L})$ and $D\left(\mathbf{L}^{(*)}\right)$ by (3.5). Let $\sigma(u)$ be compact. Then regarded as a function of $x_{0}, x_{0} \leqq 0$, with values in $H_{0}^{-k-1}\left(E^{p-1}\right), D_{0}^{k} u$ lies in $\mathfrak{L}_{2}\left(x_{0} \leqq 0, H_{0}^{-k-1}\left(E^{p-1}\right)\right.$ ) and is continuous (after correction on a set of measure zero). Moreover, as such a function,

$$
D_{0}^{k} u_{i}(0)=0, \quad r<i \leqq m .
$$

By $E_{-}$we mean $\left\{x_{0}: x_{0} \leqq 0\right\}$. To interpret the lemma, recall that $\mathscr{L}_{2}(S)$ is naturally isometrically isomorphic to $\mathfrak{L}_{2}\left(E_{-}, \mathfrak{L}_{2}\left(E^{p-1}\right)\right)$. Now $D_{0}^{k} u \varepsilon \mathfrak{L}_{2}(S)$, so $D_{0}^{k} u \varepsilon \mathfrak{L}_{2}\left(E_{-}, \mathfrak{L}_{2}\left(E^{p-1}\right)\right)$ has a natural meaning. However, $H_{0}^{-k-1}\left(E^{p-1}\right) \supset \mathscr{L}_{2}\left(E^{p-1}\right)$ is a topological inclusion so $\mathfrak{L}_{2}\left(E_{-}, \mathfrak{L}_{2}\left(E^{p-1}\right)\right) \subset \mathscr{L}_{2}\left(E_{-}, H_{0}^{-k-1}\left(E^{p-1}\right)\right)$ is also a topological inclusion, and so $D_{0}^{k} u$ has meaning as an element of $\mathfrak{L}_{2}\left(E_{-}, H_{0}^{-k-1}\left(E^{p-1}\right)\right)$. The proof of this lemma is postponed until the end of this section. We use it below to complete the proof of Theorem 3.1.

As noted above, the mollifier $J_{\epsilon}$ does not leave $H_{0}^{k}(S)$ invariant, and certainly will not preserve $D(\mathrm{~L})$. We introduce a tangential mollifier to take its place. Let $\chi \varepsilon C_{0}^{\infty}\left(E^{p-1}\right)$ be a real-valued function. We define $\chi_{T} * \varphi$ for $\varphi \varepsilon \mathscr{L}_{2}(S)$ by

$$
\chi_{T} * \varphi\left(x_{0}, x\right)=\int_{E^{p-1}} \chi\left(x-x^{\prime}\right) \varphi\left(x_{0}, x^{\prime}\right) d x^{\prime} .
$$


If $f \varepsilon D^{\prime}(S)$ we define $\chi_{T} * f \varepsilon D^{\prime}(S)$ by

$$
\left(\chi_{T} * f, \varphi\right)=\left(f, \check{\chi}_{T} * \varphi\right), \quad\left(\varphi \varepsilon C_{0}^{\infty}(S)\right),
$$

with $\check{\chi}(x)=\chi(-x)$. We have for $f \varepsilon D^{\prime}(S), \alpha=\left(\alpha_{0}, \delta\right), D^{\alpha}\left(\chi_{T} * f\right)=$ $\left(D^{\delta} \chi\right)_{T} *\left(D_{0}^{\alpha} \circ f\right)=\chi_{T} *\left(D^{\alpha} f\right)$. Let $\chi_{\epsilon}(x)=\epsilon^{-p+1} \chi(x / \epsilon), x \varepsilon E^{p-1}$. Let $j$ be a realvalued function with the properties (2.5). We now fix $\chi$ by setting $\chi\left(x_{1}, \cdots, x_{p-1}\right)$ $=j\left(x_{1}\right) \cdots j\left(x_{p-1}\right)$ and define the tangential mollifier $J_{\epsilon}$ by

$$
J_{\epsilon T} f=\chi_{\epsilon T} * f, \quad\left(f \varepsilon D^{\prime}(S)\right) .
$$

We have, parallel to Lemma 2.6 and Corollary 2.4,

Lemma 3.8. If $j$ is any integer and $v \varepsilon H_{0}^{i}(S)$, then $v_{\varepsilon}=J_{\mathrm{\epsilon} T} v \varepsilon H_{0}^{i}(S)$ and $\left\|v_{\epsilon}\right\|_{i} \leqq\|v\|_{i}$. Moreover $\lim _{\epsilon \rightarrow 0}\left\|v_{\epsilon}-v\right\|_{i}=0$.

Lemma 3.9. Let $\varphi \varepsilon H_{0}^{k}(S)$. Let $A$ be an $m \times m$ matrix, $A \varepsilon C_{b}^{|\alpha|}(S)$, where $\alpha=\left(\alpha_{0}, \delta\right),|\alpha| \leqq 2 k+1, \alpha_{0}<2 k+1$. Then $D^{\alpha}\left(A J_{\epsilon T} \varphi\right)$ and $D^{\alpha} J_{\epsilon T}(A \varphi)$ are in $H_{0}^{-k}(S)$. There exists $a c, c \geqq 0$, independent of $\varphi \varepsilon H_{0}^{k}(S)$ and $\epsilon, 0<\epsilon<1$, such that

(i)

$$
\left\|D^{\alpha}\left(A J_{\epsilon T} \varphi-J_{\epsilon T}(A \varphi)\right)\right\|_{-k} \leqq c\|\varphi\|_{k}
$$

Moreover

$$
\lim _{\epsilon \rightarrow 0}\left\|D^{\alpha}\left(A J_{\epsilon T} \varphi-J_{\epsilon T}(A \varphi)\right)\right\|_{-k}=0 .
$$

Proofs follow standard lines and are omitted.

Corollary 3.9. (Corollary to Lemma 3.6). Let $u$ satisfy the assumptions of Lemma 3.6 and $u_{\epsilon}=J_{\epsilon} u$. Then $D^{\alpha} u_{\epsilon}$ is continuous as a function on $\left\{\left(x_{0}, x\right)\right.$ : $\left.x_{0} \leqq 0, x \varepsilon E^{p-1}\right\}$ for $\alpha=\left(\alpha_{0}, \delta\right)$ and $\alpha_{0} \leqq k$. Moreover $D_{0}^{i} u_{\epsilon}(0, x)=0$ for $0 \leqq$ $j \leqq k-1$, and $D_{0}^{k} u_{\epsilon}(0, x) \varepsilon N(x), x \varepsilon E^{p-1}$.

Proof. Let $\alpha=\left(\alpha_{0}, \delta\right)$ where $\alpha_{0}=k$. (If $\alpha_{0}<k, D^{\alpha} u_{\epsilon}$ may be obtained from $D_{0}^{k} D^{\delta} u_{\epsilon}$ by integration with respect to $\left.x_{0}.\right)$ We have $D^{\alpha} u_{\epsilon}=\left(D^{\delta} \chi_{\epsilon}\right)_{T} * D_{0}^{\alpha} u_{\text {, }}$ so for $x_{0}^{\prime} \leqq 0$ and $x_{0} \leqq 0$,

$$
\begin{aligned}
\left|D^{\alpha} u_{\epsilon}\left(x_{0}, x\right)-D^{\alpha} u_{\epsilon}\left(x_{0}^{\prime}, x^{\prime}\right)\right| \leqq & \left|\int_{E^{p-2}} \kappa_{x}\left(x^{\prime \prime}\right)\left(D_{0}^{k} u\left(x_{0}, x^{\prime \prime}\right)-D_{0}^{k} u\left(x_{0}^{\prime}, x^{\prime \prime}\right)\right) d x^{\prime \prime}\right| \\
& +\left|\int_{E^{p-1}}\left(\kappa_{x}\left(x^{\prime \prime}\right)-\kappa_{x^{\prime}}\left(x^{\prime \prime}\right)\right) D_{0}^{k} u\left(x_{0}^{\prime}, x^{\prime \prime}\right) d x^{\prime \prime}\right|,
\end{aligned}
$$

where $\kappa_{x}\left(x^{\prime \prime}\right)=D^{\delta} \chi_{\epsilon}\left(x-x^{\prime \prime}\right)$. As an element of $H_{0}^{k+1}\left(E^{p-1}\right)$ (in the scalar case) $\kappa_{x}$ is continuous and bounded uniformly in $x$. We apply the Schwartz inequality to above estimate and get

$$
\begin{aligned}
\left|D^{\alpha} u_{\epsilon}\left(x_{0}, x\right)-D^{\alpha} u\left(x_{0}^{\prime}, x^{\prime}\right)\right| \leqq & \left\|\kappa_{x}\right\|_{k+1, E^{p-1}}\left\|D_{0}^{k} u\left(x_{0}^{\prime}\right)-D_{0}^{k} u\left(x_{0}\right)\right\|_{-k-1, E^{p-1}} \\
& +\left\|\kappa_{x}-\kappa_{x}^{\prime}\right\|_{k+1, E^{p-1}}\left\|D_{0}^{k} u\left(x_{0}^{\prime}\right)\right\|_{-k-1, E^{p-1}} .
\end{aligned}
$$

The corollary follows from this estimate and the assertions of Lemma 3.6. 
Now assume we know $u_{\epsilon}=J_{\epsilon T} u \varepsilon D\left(\mathrm{~L}_{\varepsilon}\right)$. Then

$$
\mathrm{L}_{\mathrm{s}} u_{\mathrm{\epsilon}}=\mathrm{L}_{w} u_{\mathrm{e}}=\left(D_{0}^{2 k+1}+M\right) J_{\mathrm{e} T} u
$$

where

$$
L=D_{0}^{2 k+1}+\sum_{\alpha_{0}<2 k+1} A_{\alpha} D^{\alpha}=D_{0}^{2 k+1}+M .
$$

Hence $\mathrm{L}_{s} u_{\epsilon}=\left(\mathrm{L}_{w} J_{\epsilon T}-J_{\epsilon T} \mathrm{~L}_{w}\right) u+J_{\epsilon T} \mathrm{~L}_{w} u$ because $u \varepsilon D\left(\mathrm{~L}_{w}\right)$ by assumption.

Lemma 3.10. $J_{\epsilon T}$ leaves $D\left(\mathrm{~L}_{w}\right)$ invariant. The operators $T_{\mathrm{e}}=\left[\mathrm{L}_{w}, J_{e T}\right]$ with $D\left(T_{\epsilon}\right)=D\left(\mathbf{L}_{w}\right)$ and range in $H_{0}^{-k}(S)$ are bounded uniformly in $\epsilon, 0<\epsilon<1$. The operators $T_{\mathrm{\epsilon}}$ have strong limit zero as $\epsilon \rightarrow 0$.

Proof. Let $v \varepsilon D\left(\mathrm{~L}_{w}\right)$, i.e., there is a $g \varepsilon H_{0}^{-k}(S)$ such that $\left(v, \mathrm{~L}^{(*)} \varphi\right)=(g, \varphi)$ for all $\varphi \varepsilon D\left(L^{(*)}\right)$. Then

$$
\left(J_{\epsilon T} v, \mathbf{L}^{(*)} \varphi\right)=\left(v,\left(J_{\epsilon T} L^{(*)}-\mathbf{L}^{(*)} J_{\epsilon T}\right) \varphi\right)+\left(J_{\epsilon T} g, \varphi\right)
$$

for all $\varphi \varepsilon D\left(\mathrm{~L}^{(*)}\right)$, for clearly $J_{\epsilon T}$ leaves $D\left(\mathrm{~L}^{(*)}\right)$ invariant. From Lemma 3.9 it follows that $\varphi \rightarrow\left(J_{\epsilon T} L^{(*)}-L^{(*)} J_{\epsilon T}\right) \varphi, \varphi \varepsilon D\left(L^{(*)}\right)$ is bounded uniformly in $\epsilon, 0<\epsilon<1$, as a map of $D\left(\mathrm{~L}^{(*)}\right)$ into $H_{0}^{-k}(S)$. This depends on the fact that

$$
\left(J_{\epsilon T} L^{(*)}-L^{(*)} J_{\epsilon T}\right) \varphi=\left(J_{\epsilon T} M^{(*)}-M^{(*)} J_{\epsilon T}\right) \varphi
$$

for $\varphi \in D\left(\mathrm{~L}^{(*)}\right)$, i.e., that $\left[D_{0}^{2 k+1}, J_{\epsilon T}\right]=0$. Equation (3.11) says, therefore, that $J_{\epsilon T} v \varepsilon D\left(\mathrm{~L}_{w}\right)$ and $\mathrm{L}_{w} J_{\epsilon T} v=A_{\epsilon}^{*} v+J_{\epsilon T} \mathrm{~L}_{w} v$, where $A_{\epsilon}=J_{\epsilon T} \mathrm{~L}^{(*)}-\mathrm{L}^{(*)} J_{\epsilon T}$, $D\left(A_{\epsilon}\right)=D\left(\mathrm{~L}^{(*)}\right)$. Hence $T_{\epsilon}=A_{\epsilon}^{*}$ on $D\left(\mathrm{~L}_{w}\right)$. The last assertion of the lemma follows from the uniform boundedness of $T_{\epsilon}$ in $\epsilon$ and the fact that $\lim _{\epsilon \rightarrow 0}\left\|T_{\epsilon} \psi\right\|_{-k}$ $=0$ for $\psi \varepsilon C_{0}^{\infty}(S)$.

Using Lemmas 3.8 and 3.10 we find

$$
\begin{aligned}
& \lim _{\epsilon \rightarrow 0}\left(\left\|\mathbf{L}_{s} u_{\epsilon}-\mathbf{L}_{w} u\right\|_{-k}+\left\|u_{\epsilon}-u\right\|_{k}\right) \\
& \leqq \lim _{\epsilon \rightarrow 0}\left(\left\|\left(\mathbf{L}_{w} J_{\epsilon T}-J_{\epsilon T} \mathbf{L}_{w}\right) u\right\|_{-k}+\left\|J_{\epsilon T} \mathbf{L}_{w} u-\mathbf{L}_{w} u\right\|_{-k}+\left\|J_{\epsilon T} u-u\right\|_{k}\right)=0,
\end{aligned}
$$

so $\left\{u, \mathbf{L}_{w} u\right\}$ is in the closure of the graph of $\mathbf{L}$ if $\left\{u_{\epsilon}, \mathbf{L}_{w} u_{\epsilon}\right\}$ is for $0<\epsilon<1$.

To complete the proof of Theorem 3.1 it remains to prove Lemma 3.6 and also that $u_{\epsilon} \varepsilon D\left(\mathrm{~L}_{s}\right), 0<\epsilon<1$. We prove the latter statement first.

For $\varphi \varepsilon \&_{2}\left(E^{p}\right)$ let $\tau_{\gamma} \varphi \varepsilon \&_{2}\left(E^{p}\right),-1<\gamma<1$, be defined by $\tau_{\gamma} \varphi\left(x_{0}, x\right)=$ $\varphi\left(x_{0}+\gamma, x\right)$. Let $j$ be a function with the properties (2.5). The normal mollifier $J_{\epsilon n}, 0<\epsilon<1$, is defined on $\mathfrak{L}_{2}\left(E^{D}\right)$ by

$$
J_{\epsilon n} \varphi\left(x_{0}, x\right)=\int_{-\infty}^{\infty} \epsilon^{-1} j\left(\left(x_{0}-x_{0}^{\prime}\right) / \epsilon\right) \varphi\left(x_{0}^{\prime}, x\right) d x_{0}^{\prime} .
$$

Let $v$ be any function with the properties claimed for $u_{\epsilon}$ above, i.e., $v \varepsilon D\left(\mathbf{L}_{w}\right)$, $v$ has compact support, $D^{\alpha} v$ is continuous for $\alpha=\left(\alpha_{0}, \delta\right), \alpha_{0} \leqq k, D_{0}^{i} v(0, x)=0$ 
for $0 \leqq j \leqq k-1$, and $D_{0}^{k} v(0, x) \varepsilon N(x)$. We define $v_{\gamma}, 0<\gamma<1$, by

$$
\begin{aligned}
\text { (i) } v_{\gamma} & =v_{1 \gamma}-v_{2 \gamma} \\
\text { (ii) } v_{1 \gamma} & =\tau_{-\gamma} J_{\gamma n} v \\
\text { (iii) } v_{2 \gamma} & \left(x_{0}, x\right) \\
& =h\left(x_{0}\right) \cdot\left(\sum_{0 \leqq i \leqq k-1}\left(x_{0}^{i} / j !\right) D_{0}^{i} v_{1 \gamma}(0, x)+K\left(x_{0}^{k} / k !\right) D_{0}^{k} v_{1 \gamma}(0, x)\right),
\end{aligned}
$$

where $h$ is a real-valued function, $h \varepsilon C_{0}^{\infty}\left(E^{1}\right)$, which is identically 1 in some neighborhood of $x_{0}=0$, and $K$ is the diagonal $m \times m$ matrix with 0 in the first $r$ places on the diagonal and 1 in the last $m-r$ places. We have $v_{\gamma} \varepsilon C_{0}^{\infty}\left(E^{p}\right)$ and the restriction to $S$ of $v_{\gamma}$ lies in $D(\mathrm{~L})$. We show

$$
\lim _{\gamma \rightarrow 0}\left\|v_{\gamma}-v\right\|_{k, s}+\left\|\mathbf{L} v_{\gamma}-L_{w} v\right\|_{-k, s}=0 .
$$

When $v$ is extended to $E^{p}$ by setting it equal to zero off $S$, we have $D^{\alpha} v$ is continuous for $\alpha=\left(\alpha_{0}, \delta\right), \alpha_{0}<k$ and $D^{\alpha} v(0, x)=0$ for such $\alpha$. Also $D^{\alpha} K v$ is continuous for $\alpha=\left(\alpha_{0}, \delta\right), \alpha_{0}=k$ and $D^{\alpha} K v(0, x)=0$ for such $\alpha$. It follows that $v_{2 \gamma}$ tends to zero in $\mathfrak{D}\left(E^{v}\right)$ as $\gamma \rightarrow 0$, so $v_{\gamma}$ tends to $v$ in $H_{0}^{k}(S)$ if $v_{1 \gamma}$ tends to $v$ in $H_{0}^{k}\left(E^{p}\right)$ as $\gamma \rightarrow 0$. Moreover

$$
\mathrm{L} v_{\gamma}=L v_{1 \gamma}-L v_{2 \gamma} \quad \text { (restricted to } S \text { ) }
$$

tends if $\mathrm{L}_{W} v$ in $H_{0}^{-k}(S)$ if $L v_{1 \gamma}$ (restricted to $S$ ) tends to $\mathrm{L}_{w} v$ in $H_{0}^{-k}(S)$. Now $\tau_{-\gamma}$ and $J_{\gamma n}$ both tend strongly to the identity in $H_{0}^{k}\left(E^{p}\right)$ as $\gamma \rightarrow 0$, so $\lim _{\gamma \rightarrow 0}\left\|v_{\gamma}-v\right\|_{k, s}=0$. Now we show $L v_{1 \gamma}$ tends to $\mathrm{L}_{w} v$ in $H_{0}^{-k}(S)$.

Let $\psi \varepsilon C_{0}^{\infty}(S)$. We have

$$
\left(L v_{1 \gamma}, \psi\right)=\left(\mathrm{L}_{w} v, J_{\gamma n} \tau_{\gamma} \psi\right)+\left(v,\left(\tau_{\gamma} J_{\gamma n} L^{(*)}-L^{(*)} J_{\gamma n} \tau_{\gamma}\right) \psi\right)
$$

and

$$
\tau_{\gamma} J_{\gamma_{n}} L^{(*)}-L^{(*)} J_{\gamma_{n}} \tau_{\gamma}=\sum_{\alpha_{0}<2 k+1}(-1)^{|\alpha|} D^{\alpha}\left(\tau_{\gamma} J_{\gamma_{n}} A_{\alpha}^{\prime}-A_{\alpha}^{\prime} J_{\gamma_{n}} \tau_{\gamma}\right),
$$

since the coefficient of $D_{0}^{2 k+1}$ in $L$ is the identity matrix. One now plugs (3.14) into (3.13) and considers each term due to the commutator (3.14) on the right in (3.13). In terms where $|\alpha|=2 k+1$ integrate once by parts, throwing one differentiation in a tangential direction on $v$. In this way we express $L v_{1 \gamma}-$ $\left(J_{\gamma n} \tau_{\gamma}\right)^{*} \mathrm{~L}_{w} v$ (where $J_{\gamma_{n} \tau_{\gamma}}$ is regarded as a map of $H_{0}^{k}(S)$ into itself) as a sum of terms of the form $T_{\gamma \alpha}^{*} D_{i} v$ and $T_{\gamma \alpha}^{*} v$ where $1 \leqq i \leqq p-1$ and $T_{\gamma \alpha}: H_{0}^{k}(S) \rightarrow$ $H_{0}^{-k}(S)$ is given by an expression of the form

$$
T_{\gamma \alpha} \psi=D^{\alpha}\left(\tau_{\gamma} J_{\gamma n} B-B J_{\gamma n} \tau_{\gamma}\right) \psi
$$

with $|\alpha| \leqq 2 k, B \varepsilon C_{b}^{|\alpha|}(\bar{S})$. It is not hard to see that $\left(T_{\gamma \alpha}\right)^{*}$ tends to zero strongly with $\gamma$ and $\left(J_{\gamma n} \tau_{\gamma}\right)$ tends strongly to the identity as $\gamma \rightarrow 0$. Thus $\lim _{\gamma \rightarrow 0}\left\|L v_{1 \gamma}-\mathrm{L}_{w} v\right\|_{-k}=0$. It remains to prove Lemma 3.6, which we do now. Let $u \varepsilon D\left(\mathbf{L}_{w}\right)$ and $\sigma(u)$ be contained in the slab $-\frac{1}{2} \leqq x_{0} \leqq 0$. Let $w \varepsilon H_{0}^{k}(S)$ 
be such that

$$
\left(u, \mathbf{L}^{(*)} v\right)=\left(\mathbf{L}_{w} u, v\right)=(w, v)_{k} \quad\left(v \varepsilon D\left(\mathbf{L}^{(*)}\right)\right)
$$

We rewrite (3.15) as

$$
\begin{aligned}
\int_{-\infty}^{0} \int_{E^{p-1}}\left(\sum _ { | \alpha | \leqq k } ( D ^ { \alpha } w ( x _ { 0 } , x ) ) \cdot \left(D^{\alpha} v\left(x_{0}, x\right)\right.\right. \\
\left.-u\left(x_{0}, x\right) \cdot L^{(*)} v\left(x_{0}, x\right)\right) d x_{0} d x=0, \quad\left(v \varepsilon D\left(\mathbf{L}^{(*)}\right)\right) .
\end{aligned}
$$

Let $\mathcal{O}$ be a real-valued function, $\mathcal{O} \varepsilon C_{0}^{\infty}(E)$, such that $\mathcal{O}\left(x_{0}\right)=1$ for $1 \geqq$ $x_{0} \geqq-\frac{3}{4}$ and $\Theta\left(x_{0}\right)=0$ for $-1 \geqq x_{0}$. We define, for $y \leqq 0$ and $h \varepsilon C_{0}^{\infty}\left(E^{p-1}\right)$,

$$
\begin{aligned}
T_{\nu}(h)=\int_{-1}^{y} \int_{E^{p-1}}\left(\sum_{|\alpha| \leqq k}\right. & \left(\left(D^{\alpha} w\right) \cdot\left(D^{\alpha}\left(y-x_{0}\right)^{k} \mathcal{O}\left(x_{0}\right) h(x)\right)\right) \\
& \left.-u\left(x_{0}, x\right) \cdot\left(L^{(*)}\left(y-x_{0}\right)^{k} \mathcal{O}\left(x_{0}\right) h(x)\right)\right) d x d x_{0} .
\end{aligned}
$$

One sees, after some integrations by parts,

$$
\left|T_{y}(h)\right| \leqq \text { cons. }\|h\|_{k+1, E^{p-1}}
$$

with a constant independent of $h \varepsilon C_{0}^{\infty}\left(E^{p-1}\right)$ and $y$. Moreover, due to the way $T_{y}(h)$ depends on $y$,

$$
\mid T_{a}(h)-T_{b}(h) \leqq \text { const. }|a-b|^{1 / 2}\|h\|_{k+1, E^{p-1}} .
$$

For the present we denote by $[\varphi, \psi]$ and $[\psi, \varphi]$ the natural pairings of $\varphi \varepsilon H_{0}^{k+1}\left(E^{p-1}\right)$ and $\psi \varepsilon H_{0}^{-k-1}\left(E^{p-1}\right)$. We define $f \varepsilon \mathcal{L}_{2}\left(E_{-}, H_{0}^{-k-1}\left(E^{p-1}\right)\right)$ by setting

$$
[f(y), h]=T_{y}(h), \quad\left(h \varepsilon C_{0}^{\infty}\left(E^{p-1}\right), y \varepsilon E_{-}\right) .
$$

Note that $f$ has support in $[-1,0]$ and is continuous by (3.19). A simple relation between $D_{0}^{k} u$ and the continuous function $f$, as elements of $\mathcal{L}_{2}\left(E_{-}, H_{0}^{-k-1}\left(E^{p-1}\right)\right)$, will be found. Let $\eta \varepsilon C_{0}^{\infty}(S)$. Using the definitions (3.17) and (3.20), a calculation yields

$$
\begin{aligned}
\int_{-1}^{0} T_{y}(\eta(y)) d y=\int_{-1}^{0}[f(y), \eta(y)] d y & \\
= & \int_{-1}^{0} \int_{E^{p-1}}\left(\sum_{|\alpha| \leqq k}\left(D^{\alpha} w\left(x_{0}, x\right)\right) \cdot \int_{x_{0}}^{0} D^{\alpha}\left(y-x_{0}\right)^{k} \mathcal{O}\left(x_{0}\right) \eta(y, x) d y\right. \\
& \left.-u\left(x_{0}, x\right) \cdot \int_{x_{0}}^{0} L^{(*)}\left(\left(y-x_{0}\right)^{k} \mathcal{O}\left(x_{0}\right) \eta(y, x) d y\right)\right) d x d x_{0} .
\end{aligned}
$$

In the left terms of this equation $\eta$ is regarded as a function mapping $y \in E_{-}$ into $C_{0}^{\infty}\left(E^{p-1}\right) \subset H_{0}^{k+1}\left(E^{p-1}\right)$. Set

$$
g\left(x_{0}, x\right)=\int_{x_{0}}^{0}\left(\left(y-x_{0}\right)^{k} \mathcal{O}\left(x_{0}\right) \eta(y, x) d y .\right.
$$


In the slab $-\frac{3}{4} \leqq x_{0} \leqq 0, \mathcal{O}\left(x_{0}\right)=1$ and we have

$$
\begin{aligned}
L^{(*)} g-\int_{x_{0}}^{0} L^{(*)}\left(\left(y-x_{0}\right)^{k} \mathcal{O}\left(x_{0}\right) \eta(y, x)\right) d y \\
=\sum_{\alpha_{0}>k}(-1)^{|\alpha|+k+1} k ! D_{0}^{\alpha_{0}-k-1} D^{\delta}\left(A_{\alpha}^{\prime}\left(x_{0}, x\right) \eta\left(x_{0}, x\right)\right)
\end{aligned}
$$

in this slab, which contains $\sigma(u)$. Hence (3.21) may be written

$$
\begin{aligned}
& \int_{-1}^{0} {[f(y), \eta(y)] d y=\int_{-1}^{0} \int_{E^{p-1}}\left(\sum_{|\alpha| \leqslant k}\left(D^{\alpha} w\left(x_{0}, x\right)\right) \cdot\left(D^{\alpha} g\right)\right.} \\
&\left.-u\left(x_{0}, x\right) \cdot L^{(*)} g\right) d x d x_{0} \\
& \quad+\int_{-1}^{0} \int_{E^{p-1}} u\left(x_{0}, x\right) \cdot \sum_{\alpha_{0}>k}(-1)^{|\alpha|+k+1} k ! D_{0}^{\alpha_{0}-k-1} D^{\delta}\left(A_{\alpha}^{\prime} \eta\right) d x d x_{0} .
\end{aligned}
$$

Now $g\left(x_{0}, x\right)=\int_{x_{0}}^{0}\left(y-x_{0}\right)^{k} \mathcal{O}\left(x_{0}\right) \eta(y, x) d y$ is an element of $D\left(\mathbf{L}^{(*)}\right)$ since $g$ and its first $k$ derivatives vanish at $x_{0}=0$. Now, via (3.16), (3.23) may be written

$$
\begin{aligned}
\int_{-1}^{0} \int_{E^{p-1}}( & \left.D_{0}^{k} u\left(x_{0}, x\right)\right) \cdot \eta\left(x_{0}, x\right) d x d x_{0} \\
= & (1 / k !) \int_{-1}^{0}[f(y), \eta(y)] d y \\
& -\int_{-1}^{0} \int_{E^{p-1}} \sum_{2 k+1>\alpha_{0}>k}(-1)^{|\alpha|}\left(D^{\alpha_{0}-k-1} u\right) \cdot D^{\delta}\left(A_{\alpha}^{\prime} \eta\right) d x d x_{0},
\end{aligned}
$$

where several integrations by parts and the fact that the coefficient of $D_{0}^{2 k+1}$ in $L$ is the identity have been used. Since $u \varepsilon H_{0}^{k}(S)$, if $0 \leqq j<k$ then $D_{0}^{j} u\left(x_{0}, x\right)$ $=\int_{0}^{x_{0}} D_{0}^{j+1} u(s, x) d s$. It follows that $f_{\alpha} \varepsilon L_{2}\left(E_{-}, H_{0}^{-k-1}\left(E^{p-1}\right)\right)$ defined by

$$
\left[f_{\alpha}(y), h\right]=\int_{E^{p-1}}\left(D_{0}^{\alpha_{0}-k-1} u(y, x)\right) \cdot D^{\delta}\left(A_{\alpha}^{\prime}(y, x) h(x)\right) d x
$$

is Hölder continuous as a function on $E_{-}$with values in $H_{0}^{-k-1}\left(E^{p-1}\right)$ and $f_{\alpha}(0)=0$ for $\alpha=\left(\alpha_{0}, \delta\right), 2 k+1>\alpha_{0}>k,|\alpha| \leqq 2 k+1$. Combining (3.24) and (3.25) we see

$$
D_{0}^{k} u=(1 / k !) f-\sum_{2 k+1>\alpha_{0}>k}(-1)^{|\alpha|} f_{\alpha}
$$

as elements of $\mathfrak{L}_{2}\left(E_{-}, H_{0}^{-k-1}\left(E^{p-1}\right)\right)$. Moreover $D_{0}^{k} u(0)=(1 / k !) f(0)$ and the last $m-r$ components of $f$ vanish (as distributions) in view of (3.16), and the definitions of $f$ and $D\left(\mathrm{~L}^{(*)}\right)$. This completes the proof of Lemma 3.6 and, with it, the proof of Theorem 3.1. 
4. The symmetric positive case. In this paragraph we prove

Theorem 4.1. Let $\Omega, L$ and $N(x)$ satisfy the conditions of Theorem I. Let $L+L^{(*)}$ be of order $2 k$ and let

$$
\left(\left(L+L^{(*)}\right) u, u\right) \geqq c\|u\|_{k}^{2} \quad\left(u \varepsilon C_{0}^{\infty}(\Omega)\right),
$$

be satisfied for some fixed $c>0$. Let $N(x)$, for $x \varepsilon \beta$, be maximal among linear subspaces of $C^{m}$ with respect to the condition

$$
\xi \cdot B(x) \xi \geqq 0 \quad(\xi \varepsilon N(x)),
$$

where $B(x)=(-1)^{k} \sum_{|\alpha|=2 k+1} A_{\alpha}(x) n(x)^{\alpha}$ for $x \varepsilon \beta(n(x)$ is the exterior normal to $\beta$ ). Then $\mathrm{L}_{s}$ is a $1-1$ map of $H_{0}^{k}(\Omega)$ onto $H_{0}^{-k}(\Omega)$.

Recall that $L=\sum A_{\alpha} D^{\alpha}$ is called symmetric positive if $A_{\alpha}=A_{\alpha}^{\prime}$ for $|\alpha|=$ $2 k+1$ and (4.2) is satisfied. Condition (4.2) is a Garding inequality for the symmetric part of $2 L$, which implies $L+L^{(*)}$ is elliptic. Conversely if $L+L^{(*)}$ is elliptic of order $2 k$ then either $L+C I$ or $-L+C I$ is symmetric positive for sufficiently large positive $C$. Any differential expression of order $2 k+1$ with symmetric coefficients in its principal part differs from a symmetric positive expression by an expression of order $2 k$.

Proof of Theorem 4. It is easy to see that the maximality of $N(x)$ with respect to (4.3) implies that $N^{*}(x)=(B(x) N(x))^{\perp}$ is non-positive for the same form (see [5, pg. 443]). Using Green's formula (1.9), we then find

$$
\begin{aligned}
(u, \mathrm{~L} u)+(\mathrm{L} u, u) & \geqq\left(\left(L+L^{(*)}\right) u, u\right) & & (u \varepsilon D(\mathrm{~L})), \\
\left(v, \mathbf{L}^{(*)} v\right)+\left(\mathbf{L}^{(*)} v, v\right) & \geqq\left(\left(L+L^{(*)}\right) v, v\right) & & \left(v \varepsilon D\left(\mathbf{L}^{*}\right)\right) .
\end{aligned}
$$

Now we use the continuity of $L+L^{(*)}$ as a map from $H_{0}^{k}(\Omega)$ into $H_{0}^{-k}(\Omega)$, (4.2), and Schwartz's inequality to deduce

$$
\left\|\mathrm{L}_{\varepsilon} u\right\|_{-k} \geqq c^{\prime}\|u\|_{k} \quad\left(u \varepsilon D\left(\mathrm{~L}_{s}\right)\right)
$$

and

$$
\left\|\mathrm{L}^{(*)} v\right\|_{-k} \geqq c^{\prime}\|v\|_{k} \quad\left(v \varepsilon D\left(\mathbf{L}^{(*)}\right)\right),
$$

where $c^{\prime}$ is some positive constant. Now (4.5) implies $L_{s}$ is $1-1$ and has closed range. If $\mathrm{L}_{s}$ is not onto $H_{0}^{-k}(\Omega)$ then there is a $v \varepsilon H_{0}^{k}(\Omega), v \neq 0$, such that

$$
\left(\mathrm{L}_{s} u, v\right)=0 \quad u \varepsilon D\left(\mathbf{L}_{s}\right) \text {. }
$$

Then $\mathbf{L}_{s}^{*} v=0$. But $\mathbf{L}_{s}=\mathbf{L}^{(*)}$, in virtue of Theorem $\mathrm{I}$, so $\mathrm{L}_{s}^{*}$ is the closure of $\mathbf{L}^{(*)}$. However, the closure of $\mathbf{L}^{(*)}$ has a trivial null space by (4.6). The theorem is established. 


\section{References}

[1] H. O. Cordes, Boundary Problems Regardless of Type, Lecture Notes, University of California at Berkeley, 1960.

[2] K. O. Friedrichs, The identity of weak and strong extensions of differential operators, Trans. Amer. Math. Soc., 55 (1944) 132-151.

[3] - Symmetric positive linear differential equations, Comm. Pure Appl. Math., 11 (1958) 333-418.

[4] K. O. Friedrichs \& P. D. Lax, Boundary value problems for first order operators, Comm. Pure Appl. Math., 18 (1965) 355-388.

[5] P. D. LAX \& R. S. PhIllips, Local boundary conditions for dissipative symmetric linear differential operators, Comm. Pure Appl. Math., 13 (1960) 427-455.

[6] R. S. Phillips \& L. Sarason, Singular symmetric positive first order differential operators, J. Math. Mech., 15 (1966) 235-272.

[7] L. Sarason, On weak and strong solutions of boundary value problems, Comm. Pure Appl. Math., 15 (1962) 237-288.

University of California at Berkeley and Stanford University Date communicated: December 27, 1967 